\title{
Study on the Formation and Properties of Liquid Crystal Emulsion in Cosmetic
}

\author{
Wanping Zhang", Lingyan Liu \\ School of Perfume and Aroma technology, Shanghai Institute of Technology, Shanghai, China. \\ Email: *zhwanp@yahoo.com.cn
}

Received January $29^{\text {th }}, 2013$; revised March $2^{\text {nd }}, 2013$; accepted March $9^{\text {th }}, 2013$

Copyright (C) 2013 Wanping Zhang, Lingyan Liu. This is an open access article distributed under the Creative Commons Attribution License, which permits unrestricted use, distribution, and reproduction in any medium, provided the original work is properly cited.

\begin{abstract}
In this paper, the formation of liquid crystal structure in preparation of emulsion and the change of those liquid crystal structures during storage and usage were studied. Besides, the rheological and moisturizing property of the liquid crystal structure emulsion was investigated as well. The results show that the liquid crystal structure at oil-water interface in the emulsion forms gradually with cooling process after homogenization. The liquid crystal structure doesn't change significantly during the storage within 12 months. And after emulsion being stored for 18 months, the crystal structure starts to decompose. Upon application on the skin, the liquid crystal structure of emulsion was found to transform into other form with rubbing, although the liquid crystal structure still remains. The rheological data shows that liquid crystal emulsion exhibits solid-like (elastic) property during storage, which is favorable for good stability. On the other hand, liquid crystal emulsion shows typical shear-thinning property upon usage, which leads to an excellent skin sensory feeling. And the improved moisturizing properties of such emulsion may be attributed to the liquid crystal structure.
\end{abstract}

Keywords: Liquid Crystal Emulsion; Rheology; Stability; Moisture

\section{Introduction}

The liquid crystal emulsion is a new type of emulsion which is different from the traditional emulsion system. It is the ordered arrangement of surfactant and oil molecules formed at the oil-water interface, and this ordered arrangement makes the emulsion of liquid crystal structure showing better application performances than conventional emulsion systems in terms of stability, controlled release and moisturizing [1-3]. The formation of liquid crystal structure in emulsion depends not only on the composition of the emulsion formulations, but also the preparation processes $[4,5]$.

The unique properties and the applications of liquid crystal emulsion have attracted many researchers in pharmaceutical and cosmetic product to do extensive studies on the special structure emulsion, including preparation and properties. Many specialty chemicals suppliers have produced emulsifiers, e.g. many non-ionic surfactants such as alkyl glycosides, polyglycerol esters, phosphates etc., for preparing emulsions with liquid crystal structure. At present, the studies of liquid crys-

${ }^{*}$ Corresponding author. talemulsion mainly focus on the theory of special systems, such as aligned structure of emulsifier molecules, stability and rheological behavior, as well as the examples of practical applications of emulsion systems $[6,7]$.

In this paper, the formation and transformation of liquid crystal structure in preparation, storage and usage were investigated. Meanwhile the rheological behavior has been studied to establish the correlation between the skin sensory characteristics and the rheology properties.

\section{Experimental Section}

\subsection{Experimental Materials and Equipments}

C16-18 APG, 99\% (Cognis); Steareth-21, 99\% (Croda); sodium stearoyl glutamate, 99\% (Cognis); Cetearyl alcohol, 99\% (Cognis); caprylic/capric triglyceride, 99\% (Cognis); stearic acid, 99\% (Cognis); Mineral oil, 99\% (Hangzhou Refinery); glycerol 99\% (Sinopharm Chemical Reagent Co., Ltd.); Dimethicone, 99\% (Dow Corning); 1,3-dihydroxy-5,5-DMH (Lonza).

FA25 High-speed shearing machine (Fluko); ECLIPSE E200 polarizing microscope (NIKON). 


\subsection{Experimental Methods}

\subsubsection{Preparation}

The formulation of emulsions comprise of oil phase, aqueous phase and other components. The oil phase includes oil and alkyl polyglycoside emulsifiers. And the aqueous phase includes deionized water and moisturizing agents. Appropriate flavors and preservatives are examples of other components. A typical formulation looks like the following: $3 \%$ emulsifier of alkyl polyglycosides, $2 \%$ stearic alcohol, $3 \%$ caprylic/capric triglyceride, $5 \%$ mineral oil, $5 \%$ dimethicone, $5 \%$ glycerin, $0.2 \%$ preservative, $0.2 \%$ flavor, $75.6 \%$ deionized water $(\mathrm{w} / \mathrm{w}$ unless otherwise indicated).

Emulsions were prepared following the typical procedures used for preparing $\mathrm{O} / \mathrm{W}$ emulsions, i.e. the aqueous phase (deionized water and moisturizing agents) was heated up to $80^{\circ} \mathrm{C}$ in a glass beaker. Meanwhile the oil phase was heated up to $80^{\circ} \mathrm{C}$ in another glass beaker. The oil phase was added to the aqueous phase followed by homogenization with F25 Ultraturrax at 13,000 rpm for 3 min. The sample was allowed to cool down to room temperature under moderate stirring at $150 \mathrm{rpm}$, and then stored at room temperature.

\subsubsection{Polarized Optical Microscope}

The samples were observed with a microscope (NIKON ECLIPSE E200). For sample preparation, a pin-tip amount of the emulsion was smeared on the microscope glass slide and then quickly covered by the cover slip. The sample was finger pressed to make it as thin as possible. A $40 \times$ objective lens and $1040 \times$ eye lens were used with cross polarizers in bright field to detect birefringence. The micrograph was taken under polarizing microscope.

\subsubsection{Rheological Measurements}

Steady-flow, thixotropy and dynamic viscoelastic properties were measured with a cone-and-plate geometry on a rheometer controlled by stress (TA Instruments Co., Ltd. AR-2000N). The cone diameter was $40 \mathrm{~mm}$. The shear rates were from 0.0001 to $200 \mathrm{~s}^{-1}$ in steady-flow and thixotropy property measurements. The dynamic viscoelasticity was measured as a function of the frequency at a small strain in the linear regions and as a function of strain at a constant frequency. The angular frequencies were from 0.1 to $100 \mathrm{~s}^{-1}$ and the strain amplitude was $0.1 \%$. The measuring temperature was $25^{\circ} \mathrm{C}$.

\subsubsection{Moisturizing Property}

The water content and the Transepidermal Water Loss (TEWL) on the skin surface were measured by Corneometer. The measurement was carried out in a room with controlled humidity $(40 \%)$ and temperature $\left(22^{\circ} \mathrm{C} \pm\right.$ $\left.1^{\circ} \mathrm{C}\right)$.

\section{Results and Discussion}

\subsection{Formation of Liquid Crystal Structure Emulsion}

Emulsions with good stability can be obtained by using a mixture of emulsifiers with preparation processes described in this paper. The formation of liquid crystal structure, however, shows strong dependence on the composition of formulation and preparation process $[6$, 7]. By optimizing the composition of formulation and preparation process, the liquid crystal emulsion can be formed. In the experiment, the formation process of the liquid crystal structure during preparation of emulsion was studied. The polarized microscope was utilized to monitor the sample during emulsification and homogenization. Photos were taken under polarized light and at $10 \mathrm{~min}, 20 \mathrm{~min}$ after homogenization and the end of the preparation, respectively.

Figure 1 shows that a small amount of liquid crystal structure was present, though disorderly, in the emulsion droplets during the emulsification and homogenization process. Photos taken 10 and 20 minutes after homogenization show increasingly higher amount of liquid crystal structure at the oil-water interface. Furthermore, the photo taken after homogenization and cooling show well developed liquid crystal structures at the interface. Therefore, it could be concluded that the liquid crystal structure of oil-water interface in the emulsion is gradually formed with cooling after homogenization.

\subsection{Stability of Liquid Crystal Structure Emulsion}

As we discussed previously, the formation of liquid crystal structure is the result of ordered arrangement of emulsifier and oil molecules at the oil-water interface. However, rearrangement may occur during the storage,
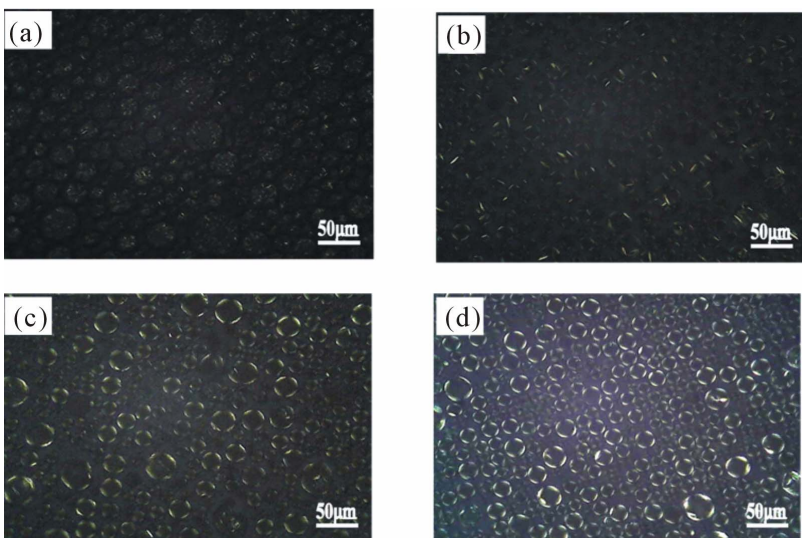

Figure 1. Liquid crystal formation during and after emulsion preparation (under polarized lighting). (a) During homogenization; (b) 10 min after homogenization; (c) $20 \mathrm{~min}$ after homogenization; (d) The final sample. 
leading to the change of liquid crystal structure, which may impact storage stability and skin feel during application. Therefore, the change of liquid crystal structure at six months, twelve months and eighteen months of storage are studied.

Figure 2 shows that liquid crystal structure doesn't change significantly during storage within 12 months, though it does seem to decompose significantly after the emulsion has been stored for 18 months. Such a phenomenon could be attributed to the fact that that the formation of liquid crystal structure strongly depends on the orderly arrangement of emulsifier and oil molecules by the way of dynamic equilibrium with the thermal motion. The thermal motion of the oil and the emulsifier molecules could reduce the ordered arrangement of the liquid crystal structure during the storage, thereby leading to the gradually decomposition of the liquid crystal structure $[8,9]$. Actually the thermal motion is favored by the presence of more liquid oils and emulsifiers. On the opposite, solid compound is not easy to move at room temperature and reduce the thermal motion. It can be concluded that increasing the content of solid compound could reduce the characteristics of thermal motion of molecules, meanwhile enhance the stability of liquid crystal structure by adjusting the formulation structure of emulsion.

\subsection{Change of the Liquid Crystal Structure Emulsion in Usage}

It is expected that the liquid crystal structure in such emulsions could lead to different application properties. To assess the relationship between the liquid crystal structure and application properties, and determine if there are perceivable benefits, it is important to study the changes of the liquid crystal structure during usage. Po-
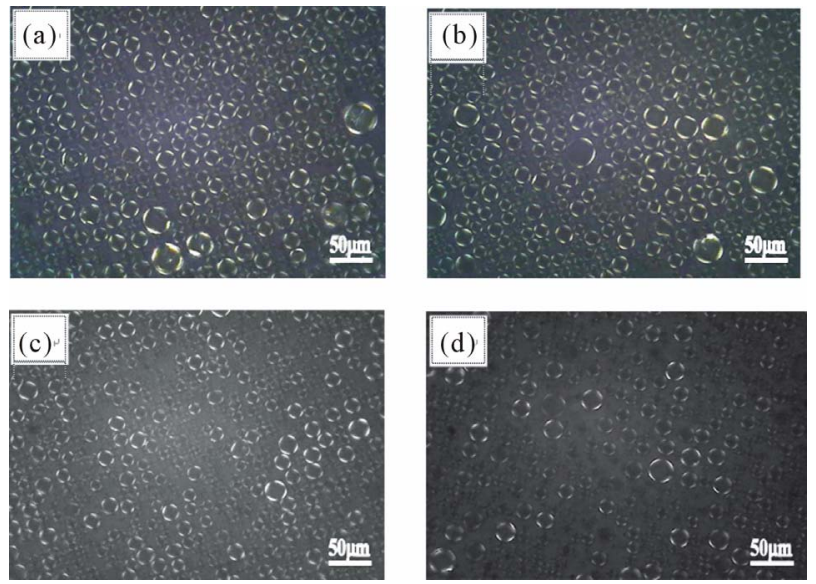

Figure 2. Liquid crystal in emulsion during storage (under polarized lighting). (a) 24 hours after preparation; (b) 6 months after preparation; (c) 12 months after preparation; (d) 18 months after preparation. larized microscope photographs were taken at different stages of usage to show the changes of the liquid crystal structure during such a process.

Figure 3 shows the significant changes of the liquid crystal structures upon rubbing onto skin. When it is just applied on the skin, the emulsion particles with liquid crystal structure still exist. With the time extending, it is conceivable that the water in the emulsion will gradually evaporate and the relative content of the oils and the emulsifiers will gradually increase, which will lead to the breakdown of the emulsion particles, and turn the liquid crystal structure with orderly distribution at oil-water interface into lamellar liquid crystal structure [8,9]. The photos (Figure 3) confirm that the liquid crystal structure of emulsion changes upon rubbing, while the liquid crystal structure still remains.

\subsection{Rheological Property of Liquid Crystal Structure Emulsion}

In theory, the liquid crystal structure should result in a few advantages than the conventional emulsion in cosmetics applications, for example, improved stability, moisture retention, controlled-release and good skin sensory feeling. In order to probe into the correlation between the liquid crystal structure and application benefits, especially stability and skin sensory feeling, the rheological properties of the liquid crystal emulsion were studied.

As shown in Figure 4, the dynamic viscosity of the liquid crystal emulsion shows Bingham behavior with shear-thinning properties. A yield stress of $31.58 \mathrm{~Pa}$ indicates good stability can be achieved during storage and transportation. Upon usage, the shear-thinning property could allow the good spreading and penetrability with finger rubbing for such emulsions. The thixotropic curve shows a little hysteresis loop that implies the recovery of
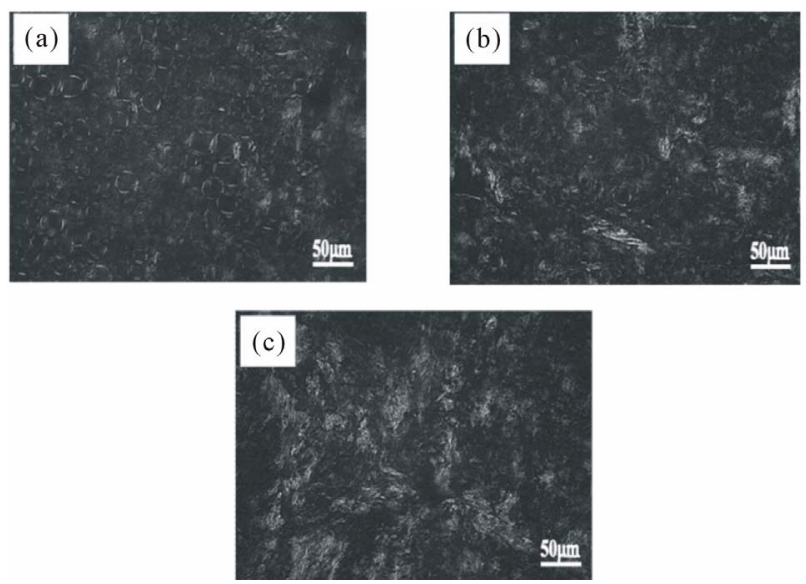

Figure 3. Liquid crystal upon application under polarized microscope. (a) Upon applying; (b) 5 min upon applying; (c) $10 \mathrm{~min}$ upon applying. 


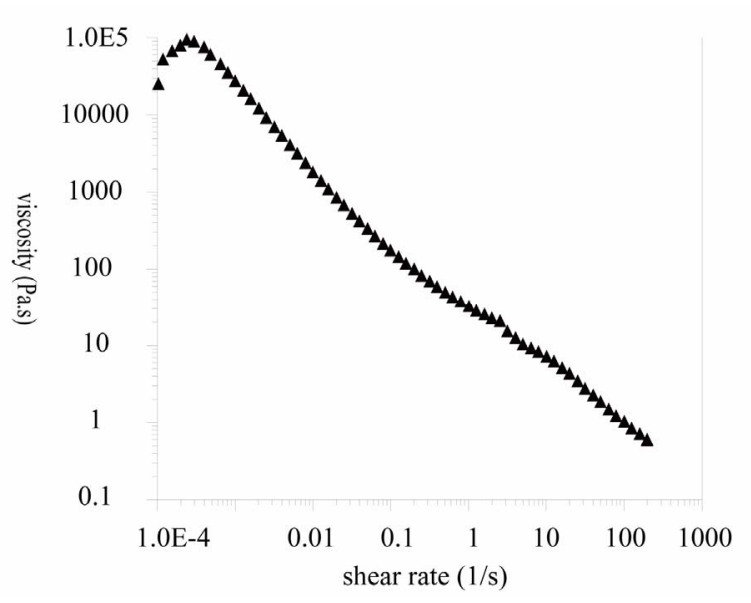

(a)

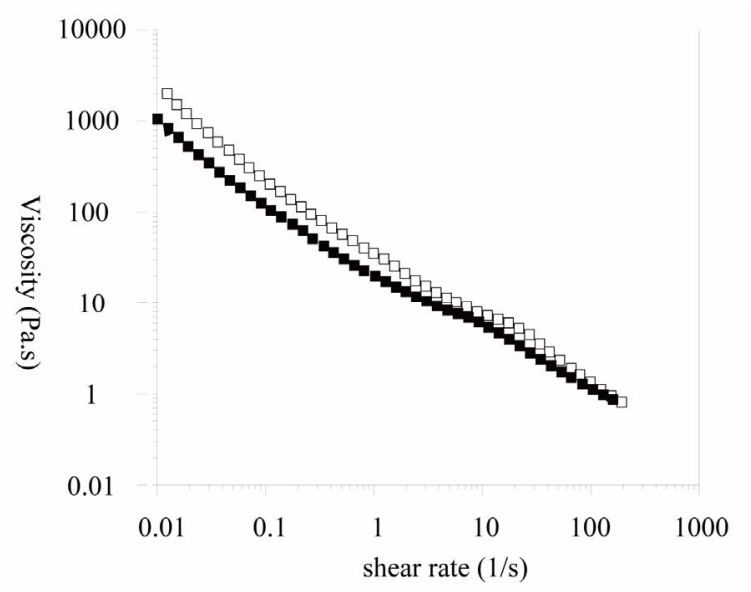

(b)

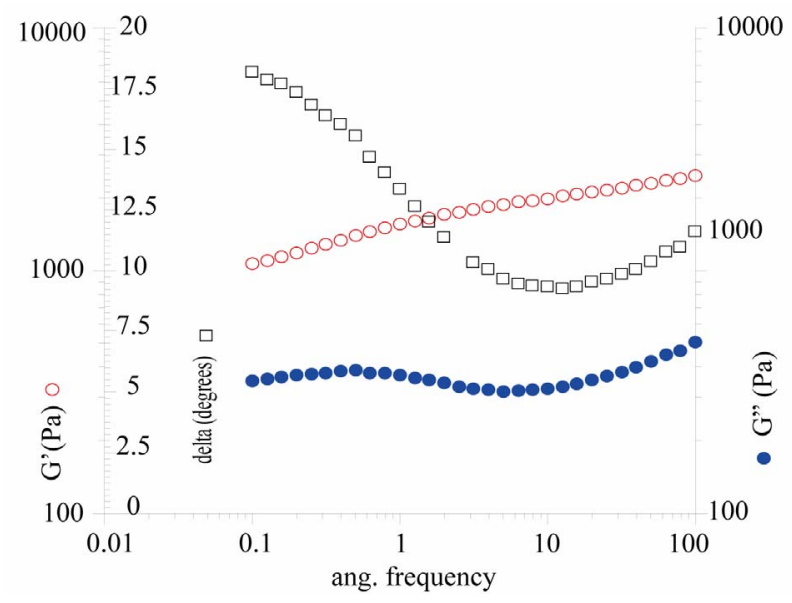

(c)

Figure 4. Rheological properties of liquid crystal structure emulsion. (a) Viscosity vs shear rate; (b) Thixotropy curve; (c) Modulus curve.

liquid crystal structure lag behind the shear stress removing. The fluidity of the liquid crystal emulsion is restored shortly after shear stress removal. The result indicates that the liquid crystal emulsion can show good spreadability during application $[10,11]$.

$\mathrm{G}^{\prime}$ is the storage modulus and $\mathrm{G}^{\prime}$ is the loss modulus. The viscoelastic curve is the G' and G" vs frequency $(\omega)$. These frequency $(\omega)$ dependence of dynamic moduli further suggest that $G$ ' response of these sample is dominant over G" response throughout entire measured $\omega$ domain, implying that solid-like (elastic) property dominates over liquid-like (viscous) property in the liquid crystal emulsion. It means that the liquid crystal emulsion exhibits solid-like (elastic) property in storage, which is good for product stability. However the shear-thinning property indicates liquid-like (viscous) property during usage, which is quite good for skin sensory feeling. Besides, G' and G" response increased with $\omega$ throughout all measured $\omega$ domain, though slightly. Such a viscoelastic response is usual for surfactant stabilized $\mathrm{O} / \mathrm{W}$ emulsion. This implies that the liquid crystal emulsion can show excellent property pertaining to emulsion used in pharmaceutical and cosmetic applications.

\subsection{Moisturizing Property of Liquid Crystal Structure Emulsion}

In order to study the influence of liquid crystal structure emulsion on moisturizing properties. The water content and the Transepidermal Water Loss (TEWL) on the skin surface, before and after the application of liquid crystal structure emulsion, were investigated by using a Corneometer.

It has been reported that the increase of C16-18 fatty alcohol in emulsions leads to increase of the formation of liquid crystal structure [6]. In these studies, we prepared emulsion samples with different content of C16-18 fatty alcohol and their respective moisturizing properties were studied.

Figure 5(a) shows that there is no change on the water content of skin surface by using the different emulsion with the different liquid crystal structure, but the different liquid crystal structure affect the Transepidermal Water Loss on the skin surface. Figure 5(b) shows that the Transepidermal Water Loss decreases with increasing the liquid crystal structure of emulsion. The good moisturizing properties of the emulsions with liquid crystal structure could be attributed to the fact that large amount of water molecules can be entrapped within the ordered, crystalline structures of the liquid crystals by association with the hydrophilic groups of the emulsifier molecules.

The latter forms ordered \& multilayer structures together with oil and other molecules at the water/oil interface of the emulsion.

\section{Conclusion}

In this paper, the formation of liquid crystal structure 


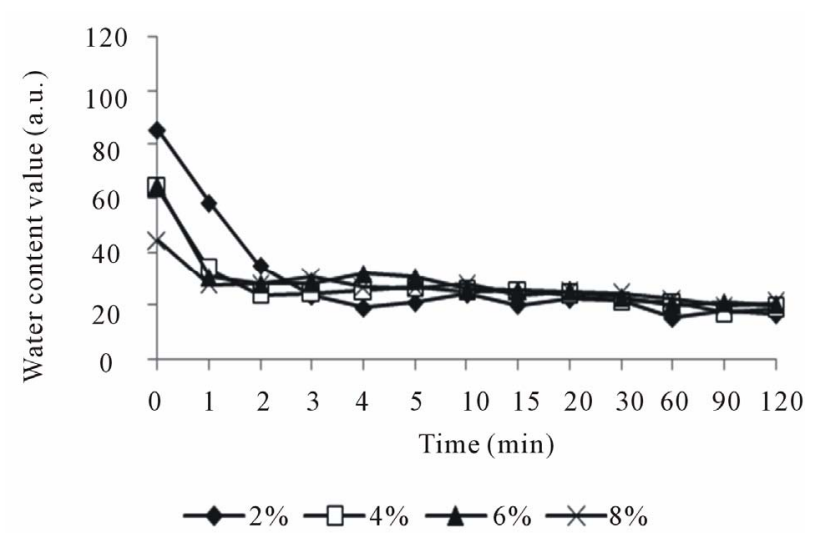

(a)

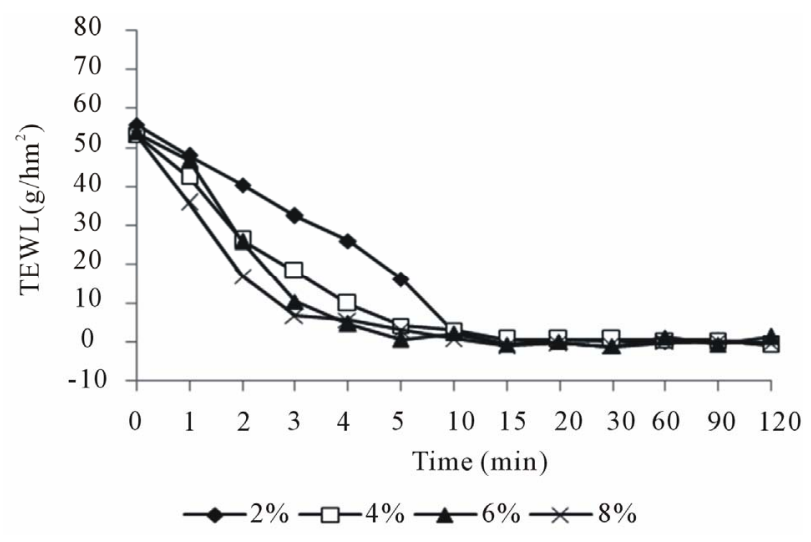

(b)

Figure 5. Moisturizing properties of liquid crystal structure emulsion. (a) Influence on the water content of skin surface; (b) Influence on the Transepidermal Water Loss on the skin surface.

during preparation of emulsion, the change of such structure during storage and cosmetic applications as well as the rheological properties were investigated. The results show that the liquid crystal structure gradually forms at the oil-water interface of the emulsion particles with cooling process after homogenization. The liquid crystal structure exists at the oil-water interfaces of emulsion particles. The liquid crystal structure doesn't change significantly during the storage within 12 months. However after being stored for 18 months, the crystal structure starts to decompose. Upon applying onto skin, the liquid crystal structure with orderly distribution at oil-water interface turns into lamellar liquid crystal structure. The liquid crystal emulsion shows solid-like (elastic) rheological properties, which can lead to good product feel, spreadability and other favorable attributes associated with cosmetic applications. The liquid crystal structure emulsion also shows good moisturizing property, which could be attributed to the entrapment of the water molecules within the emulsifier layers of the liquid crystal structure.

\section{Acknowledgements}

The authors thank Hu Jing in Shanghai Institute of Technology for establishing the methods to measure the rhology properties, Zheng Yunyun in Shanghai Research Institute of Fragrance \& Flavor Industry for support with the moisturizing properties studies.

\section{REFERENCES}

[1] Y. Liu and S. Friberg, "Role of Liquid Crystal in the Emulsification of a Gel Emulsion with High Internal Phase Fraction," Journal of Colloid and Interface Science, Vol. 340, No. 2, 2009, pp. 261-268. doi:10.1016/j.jcis.2009.08.038

[2] J. Vilasau, C. Solans, M. Gómez, et al., "Phase Behaviour of a Mixed Ionic/Nonionic Surfactant System Used to Prepare Stable Oil-in-Water Paraffin Emulsions," Colloids and Surfaces A: Physicochemical and Engineering Aspects, Vol. 384, No. 1-3, 2011, pp. 473-481. doi:10.1016/j.colsurfa.2011.05.029

[3] T. Guillaume, M. Sophie, et al., "Colloidal Shape Controlled by Molecular Adsorption at Liquid Crystal Interfaces," The Journal of Physical Chemistry B, Vol. 112, No. 14, 2008, pp. 4157-4160. doi:10.1021/jp800431y

[4] W. P. Zhang and L. Y. Liu, "Influence of Other Ingredients on the Formation of Liquid Crystal in APG System," China Surfactant Detergent \& Cosmetics, Vol. 40, No. 1, 2010, pp. 35-39.

[5] W. P. Zhang and L. L. Zhu, "Study on the Influence of Emulsion Technology on the Formation of Liquid Crystal Structural," China Surfactant Detergent \& Cosmetics, Vol. 39, No. 1, 2009, pp. 35-38.

[6] P. Saulnier, N. Anton, et al., "Liquid Crystals and Emulsions in the Formulation of Drug Carriers," Comptes Rendus Chimie, Vol. 11, No. 3, 2008, pp. 221-228. doi:10.1016/j.crci.2007.10.005

[7] T. Sonoda, Y. Takata, et al., "Effects of Emulsifiers on Crystallization Behavior of Lipid Crystals in Nanometer-Size Oil-in-Water Emulsion Droplets," Crystal Growth \& Design, Vol. 6, No. 1, 2006, pp. 306-312. doi: $10.1021 / \operatorname{cg} 050045 \mathrm{~h}$

[8] A. Bawab and S. E. Friberg, "Some Pertinent Factors in Skin Care Emulsion," Advances in Colloid and Interface Science, Vol. 123-126, 2006, pp. 313-322. doi:10.1016/j.cis.2006.06.004

[9] R. Carlos and L. Massimo, "Emulsions with Structured Continuous Phases," Current Opinion in Colloid \& Interface Science, Vol. 13, No. 4, 2008, pp. 198-205. doi:10.1016/j.cocis.2007.09.004

[10] H. M. Ribeiro, J. A. Morais, et al., "Structrre and Rheology of Semisolid O/W Creams Containing Cetyl Alcohol/ Non-Ionic Surfactant Mixed Emulsifier and Different Polymers," International Journal of Cosmetic Science, Vol. 26, No. 2, 2004, pp. 47-59. doi:10.1111/j.0412-5463.2004.00190.x

[11] M. Mohammad and A. Kenji, "Effect of Molecular Weight of Triglycerides on the Formation and Rheological 
Behavior of Cubic and Hexagonal Phase Based Gel Emulsions," Journal of Colloid and Interface Science,
Vol. 336, No. 1, 2009, pp. 329-334.

doi:10.1016/j.jcis.2009.03.054 